\title{
Why Do Firms Conduct Bi-Sourcing? ${ }^{1}$
}

\author{
Julan Du \\ Chinese University of Hong Kong \\ $\mathrm{Yi} \mathrm{Lu}$ \\ University of Hong Kong \\ Zhigang Tao \\ University of Hong Kong
}

\begin{abstract}
In acquiring the same intermediate inputs, a firm often conducts bi-sourcing, i.e., simultaneously buying from external suppliers and self-producing in an internal manufacturer. We show that the firm achieves a better bargaining position in bisourcing than in outsourcing through cross threat effect, which enhances profitability.
\end{abstract}

Key words: Bi-sourcing, Outsourcing, Cross Threat.

JEL Classification: D21, D23

\footnotetext{
${ }^{1}$ Corresponding author: Julan Du, Department of Economics, Chinese University of Hong Kong, Shatin, N.T., Hong Kong. Tel: 852-2609-8008, Fax: 852-2603-5805, Email: julandu@cuhk.edu.hk. We would like to thank Thomas Hout, David D. Li, Ping Lin, Ivan Png, Larry Qiu, Michael Riordan, Thomas Ross and participants in the 2005 Summer Workshop on Industrial Organization and Management Strategy at Tsinghua University for their helpful comments.
} 


\section{I.Introduction}

To produce outputs, a firm typically adopts outsourcing in acquiring intermediate inputs, i.e., buying components from external suppliers. However, in reality, a firm often chooses to acquire the same component by both buying from external suppliers and self-producing in an internal component manufacturer owned by the firm. We call it bi-sourcing as it contains both outsourcing and insourcing. For example, Nokia purchases a large proportion of key electronic components such as semiconductors and microprocessors from a global network of suppliers. At the same time, Nokia operates about ten manufacturing plants in nine countries to produce these components.

Why do firms conduct bi-sourcing? In outsourcing, the external component supplier keeps ownership and control over assets for upstream production. According to the property rights theory (Grossman and Hart, 1986), this can stimulate the incentive of the external supplier to improve productivity; however, it also generates the potential holdup problem in arm's-length trading relationship as the external supplier may threaten not to fulfill the contract obligations so as to capture a larger share of total surplus. Bi-sourcing can mitigate the holdup problem to a substantial extent. In negotiating with the external supplier, the firm can use the backup option of the internal supplier to minimize the holdup problem. Once holdup problem occurs, the components from the internal supplier can help avoid a halt to the production process.

At the same time, bi-sourcing can keep to a large degree the incentive of external supplier to improve productivity. The presence of an external supplier can further mitigate the internal supplier's problem of lack of incentive. As a result, the firm enjoys a better bargaining position in bi-sourcing than in outsourcing. This stimulates the firm to supply headquarter services, which in turn promotes the component supply and total output through the complementarity effect. Thus, bi-sourcing achieves a higher profitability than outsourcing does. In our example, Nokia finds that outsourcing allows it to secure inputs produced with the state-of-the-art technology, but it also involves the risk that the timely delivery of quality components may not be guaranteed. Bi-sourcing allows Nokia to strike a balance between the quality and the security of component supply.

\section{II.Model Setup}

A firm with headquarter $H$ combines headquarter services $(h)$ with component inputs $(m)$ to produce final goods. The production generates revenue function $R=f(h, m)$ that has the following characteristics: (1) $\frac{\partial f}{\partial h} \geq 0$, $\frac{\partial^{2} f}{\partial h^{2}}<0 ; \frac{\partial f}{\partial m} \geq 0, \frac{\partial^{2} f}{\partial m^{2}}<0 ;(2) f(0,)=0,. f(., 0)=0$; and $(3) \frac{\partial^{2} f}{\partial h \partial m}>$ $0, \frac{\partial^{3} f}{\partial h \partial m^{2}}<0, \frac{\partial^{3} f}{\partial h^{2} \partial m}<0$. Condition (3) indicates the complementarity between the two inputs $h$ and $m$, but the degree of complementarity decreases in both inputs. Meanwhile, each unit of both inputs requires one unit of labor to produce. For simplicity, the wage rate is normalized to 1. Moreover, the investments in $m$ and $h$ are completely specific to the trading relationship so that they have no value outside the relationship. There are three periods in the model. At time 0, $H$ chooses between outsourcing and bi-sourcing. Ex ante 
investments in input production are made at date 1 , and $m$ is supplied and combined with $h$ at date 2 . There is ex ante uncertainty about component type so that it is infeasible to make an effective long-term contract. As investments in input production are irreversible and noncontractible, the parties negotiate about the component type and price at date 2 from scratch.

\section{III.Single Outsourcing}

For simplicity, we consider one external supplier for the outsourcing scenario and call it single outsourcing. ${ }^{1}$ At date 1 , the headquarter $(H)$ signs a contract with an external supplier $M_{1}$ to purchase intermediate goods $m_{1}$, and combines it with $h$ to produce final goods and generate revenue $R_{1}=f\left(h, m_{1}\right)$. At date 2 , they bargain over the distribution of the surplus from the trading relationship by following the generalized Nash bargaining procedure. In bilateral negotiation, $H$ and $M_{1}$ have bargaining power of $\beta_{1}$ and $1-\beta_{1}$ respectively. Given that trading is efficient, Nash bargaining leads to that $H$ gets $\beta_{1} R_{1}$ and $M_{1}$ gets $\left(1-\beta_{1}\right) R_{1} . H$ and $M_{1}$ choose $h$ and $m_{1}$ to maximize $\beta_{1} R_{1}-h$ and $\left(1-\beta_{1}\right) R_{1}-m_{1}$ respectively.

\section{IV.Bi - Sourcing}

In bi-sourcing, the headquarter $(H)$ purchases the intermediate inputs from both the external $\left(M_{1}\right)$ and internal $\left(M_{2}\right)$ suppliers. Let $R=f(h, m), R_{1}=$ $f\left(h, m_{1}\right)$ and $R_{2}=f\left(h, m_{2}\right)$ denote the total revenues when both $M_{1}$ and $M_{2}$, only $M_{1}$, and only $M_{2}$ provide component inputs, respectively, where $m=$ $m_{1}+m_{2}$. Following the property rights literature, we assume that ex post bargaining occurs both under outsourcing and under insourcing, that is, $H$ negotiates with the external and internal component suppliers $\left(M_{1}\right.$ and $\left.M_{2}\right)$ respectively. The bargaining power distribution between $H$ and $M_{1}$ remains the same as before, while $H$ and $M_{2}$ have bargaining power of $\beta_{2}$ and $1-\beta_{2}$ respectively. Following Antras and Helpman (2004), we assume that $H$ has a higher bargaining power with respect to $M_{2}$ than with respect to $M_{1}$, i.e., $\beta_{2}>\beta_{1}$. This is a realistic assumption: as long as a component supplier does not have some unique production capability that can hardly be replaced, $H$ tends to have stronger bargaining power when she owns the supplier and enjoys the residual control rights or authority.

At the beginning of date $1, H$ announces the bargaining procedure, i.e., whether she will negotiate first with $M_{2}$ and then $M_{1}$ or the other way around. We consider the former case first. Using the backward deduction approach, we first look at the bargaining game between $H$ and $M_{1}$. Before that negotiation is started, $H$ has already finished the negotiation with $M_{2}$, securing a component supply of $m_{2}$ and paying $P_{2}$ to $M_{2}$. Consequently, in negotiating with $M_{1}$, $H$ expects to obtain $R-P_{1}-P_{2}$ if the trading is carried out but gets the disagreement option value $R_{2}-P_{2}$ if negotiation breaks down, whereas $M_{1}$ obtains a transfer payment $P_{1}$ from $H$ if trading is conducted and zero otherwise. As trading is efficient, Nash bargaining determines the division of social surplus

\footnotetext{
${ }^{1}$ Having two external component suppliers with differential bargaining power makes no qualitative difference to our results as long as the firm has a larger bargaining power relative to the internal supplier than the external supplier due to ownership and authority.
} 
by transfer payment $P_{1}^{*}=\left(1-\beta_{1}\right)\left(R-R_{2}\right)$. Correspondingly, $H$ obtains $R_{2}-$ $P_{2}+\beta_{1}\left(R-R_{2}\right)$ and $M_{1}$ gets $\left(1-\beta_{1}\right)\left(R-R_{2}\right)$.

Next we move back to the initial stage bargaining between $H$ and $M_{2}$. With full knowledge and rational expectations, $H$ and $M_{2}$ bargain over $R-P_{1}^{*}$. If trade with $M_{2}$ takes place, $H$ secures $R-P_{1}^{*}-P_{2}$ and $M_{2}$ obtains $P_{2}$. If there is no trade with $M_{2}, H$ can at least reap $\beta_{1} R_{1}$ from single outsourcing with $M_{1}$, while $M_{2}$ gets nothing. The Nash bargaining yields $P_{2}^{*}=\left(1-\beta_{2}\right)(R-$ $\left.P_{1}^{*}-\beta_{1} R_{1}\right)$, which can be rewritten as $P_{2}^{*}=\left(1-\beta_{2}\right)\left[\beta_{1} R+\left(1-\beta_{1}\right) R_{2}-\right.$ $\beta_{1} R_{1}$. As a result, $H, M_{1}$ and $M_{2}$ obtain profits of $\pi_{H}=R-P_{1}^{*}-P_{2}^{*}-h=$ $\beta_{1} \beta_{2} R+\beta_{1}\left(1-\beta_{2}\right) R_{1}+\beta_{2}\left(1-\beta_{1}\right) R_{2}-h, \pi_{M_{1}}=\left(1-\beta_{1}\right)\left(R-R_{2}\right)-m_{1}$, and $\pi_{M_{2}}=\left(1-\beta_{2}\right)\left[\beta_{1} R+\left(1-\beta_{1}\right) R_{2}-\beta_{1} R_{1}\right]-m_{2}$, respectively. The whole problem becomes $H, M_{1}$ and $M_{2}$ choosing $h, m_{1}$ and $m_{2}$ at date 1 to maximize $\pi_{H}, \pi_{M_{1}}$, and $\pi_{M_{2}}$, respectively.

Interestingly, we find that the sequence of bargaining does matter:

Proposition 1: For bi-sourcing to be sustainable, $H$ must negotiate with the softer party $\left(M_{2}\right)$ earlier than the tougher one $\left(M_{1}\right)$; otherwise the bi-sourcing scenario would be reduced to single outsourcing.

We know that the component supplier who is negotiated later contributes to the total revenue on top of the inputs made by the supplier that is negotiated earlier. Given the concavity of the production revenue function, the late mover always contributes less to total revenue for a given amount of inputs and in turn claims a lower marginal revenue than the early mover does. If $H$ negotiates with $M_{2}$ later than $M_{1}, M_{2}$ always encounters a lower marginal revenue than $M_{1}$ does. Since $M_{1}$, as the first mover, equates its marginal revenue with the constant marginal cost in equilibrium, $M_{2}$ will have a marginal revenue that is always lower than the marginal cost, which will depress her incentive to make investments and reduce bi-sourcing to single outsourcing.

\section{V.Choice between Bi - sourcing and Single Outsourcing}

Finally, the headquarter $H$ chooses between bi-sourcing and single outsourcing at date 0 . It turns out that bi-sourcing is more efficient.

Proposition 2: Bi-sourcing generates more profits than single outsourcing does.

In bi-sourcing, $H$ can obtain cross threat effect in negotiating with $M_{1}$ and $M_{2}$. With $M_{2}$ as a backup, $H$ can diminish the holdup problem of $M_{1}$. Moreover, with $M_{1}$ as an outside option, $H$ can also force $M_{2}$ to make relationshipspecific investments. Consequently, $H$ achieves a better bargaining position in bi-sourcing than in single outsourcing, and her supply of $h$ also increases. As $h$ and $m$ are strategic complements, this in return stimulates the component provision by $M_{1}$ and $M_{2}$, which further enhances the total profits.

\section{Conclusion}

In this paper, we provide one explanation for the superiority of bi-sourcing: the cross threat in negotiation in bi-sourcing helps the firm to mitigate the holdup problem of outsourcing, keeps the incentive of both internal and external suppliers and improves economic efficiency. 


\section{Appendix}

[Sketch of Proof to Proposition 1] As the bargaining structure is symmetric, if $H$ negotiates with $M_{1}$ earlier than $M_{2}$, we can write out the first order conditions for $H, M_{1}$ and $M_{2}$ as $\beta_{1} \beta_{2} \frac{\partial f(h, m)}{\partial h}+\beta_{1}\left(1-\beta_{2}\right) \frac{\partial f\left(h, m_{1}\right)}{\partial h}+$ $\beta_{2}\left(1-\beta_{1}\right) \frac{\partial f\left(h, m_{2}\right)}{\partial h}=1,\left(1-\beta_{1}\right)\left[\beta_{2} \frac{\partial f(h, m)}{\partial m_{1}}+\left(1-\beta_{2}\right) \frac{\partial f\left(h, m_{1}\right)}{\partial m_{1}}\right]=1$, and $(1-$ $\left.\beta_{2}\right) \frac{\partial f(h, m)}{\partial m_{2}}=1$ respectively. As $\beta_{2} \frac{\partial f(h, m)}{\partial m_{1}}+\left(1-\beta_{2}\right) \frac{\partial f\left(h, m_{1}\right)}{\partial m_{1}}>\frac{\partial f(h, m)}{\partial m_{2}}$ and $1-\beta_{1}>1-\beta_{2}$, the last two equations cannot be satisfied simultaneously. Since $M_{1}$ moves first to choose the optimal investment, the late mover $M_{2}$ always faces the situation of $\left(1-\beta_{2}\right) \frac{\partial f(h, m)}{\partial m_{2}}<1$. Thus $m_{2}=0$. It is easy to show that if $H$ negotiates with $M_{2}$ earlier than $M_{1}$, the three first order conditions can be satisfied simultaneously. QED.

[Sketch of Proof to Proposition 2] In single outsourcing, the first order conditions for $H$ and $M_{1}$ are $\beta_{1} \frac{\partial f\left(h, m_{1}\right)}{\partial h}=1$ and $\left(1-\beta_{1}\right) \frac{\partial f\left(h, m_{1}\right)}{\partial m_{1}}=1$ respectively. The former equation generates the headquarter's reaction fuction to the component supplier as $h^{S}=h^{S}\left(m ; \beta_{1}\right)$, while the latter equation generates the supplier's reaction function to the headquarter as $m^{S}=m^{S}\left(h ; \beta_{1}\right)$.

In bi-sourcing, when $H$ negotiates with $M_{2}$ earlier than with $M_{1}$, the first order conditions for $H, M_{2}$ and $M_{1}$ are $\beta_{1} \beta_{2} \frac{\partial f(h, m)}{\partial h}+\beta_{1}\left(1-\beta_{2}\right) \frac{\partial f\left(h, m_{1}\right)}{\partial h}+$ $\beta_{2}\left(1-\beta_{1}\right) \frac{\partial f\left(h, m_{2}\right)}{\partial h}=1,\left(1-\beta_{2}\right)\left[\beta_{1} \frac{\partial f(h, m)}{\partial m_{2}}+\left(1-\beta_{1}\right) \frac{\partial f\left(h, m_{2}\right)}{\partial m_{2}}\right]=1$, and $(1-$ $\left.\beta_{1}\right) \frac{\partial f\left(h, m_{1}+m_{2}\right)}{\partial m_{1}}=1$, respectively. Rewrite the equation for $H$ as $T \frac{\partial f(h, m)}{\partial h}=$ 1 , where $T=\beta_{1} \beta_{2}+\beta_{1}\left(1-\beta_{2}\right) \frac{\partial f\left(h, m_{1}\right) / \partial h}{\partial f(h, m) / \partial h}+\beta_{2}\left(1-\beta_{1}\right) \frac{\partial f\left(h, m_{2}\right) / \partial h}{\partial f(h, m) / \partial h}$. From the first order conditions for $H$ and $M_{1}$, we can obtain the reaction functions of the headquarter and the two component suppliers as $h^{B}=h^{B}(m ; T)$ and $m^{B}=m^{B}\left(h ; \beta_{1}\right)$ respectively.

The component suppliers' reaction functions coincide under bi-sourcing and single outsourcing, i.e., $m^{S}=m^{B}=g\left(h ; \beta_{1}\right)$, while the headquarter's reaction functions differ only in the exogenous variable, i.e. $h=h(m ; i), i=T$ for bi-sourcing and $i=\beta_{1}$ for single outsourcing. It is easy to show that the reaction functions $h(m ; i)$ and $g\left(h ; \beta_{1}\right)$ are increasing and concave in $m$ and $h$ respectively, and $h(m ; i)$ is increasing in the exogenous variable $i$.

Since $\frac{\partial f\left(h, m_{1}\right)}{\partial h}+\frac{\partial f\left(h, m_{2}\right)}{\partial h}>\frac{\partial f(h, m)}{\partial h}$ holds by assumption, we have $T>$ $\beta_{1}$. The intersection point of the two reaction functions under bi-sourcing lies northeast to that under single outsourcing, which means at the second-best equilibrium we have $h^{B *}>h^{S *}$, and $m^{B *}>m^{S *}$. This finally leads to higher revenue and profits under bi-sourcing than under single outsourcing. QED.

\section{Reference}

Antras, Pol and Elhanan Helpman (2004) "Global Sourcing", Journal of Political Economy, 112: 552-580.

Grossman, Sanford J. and Oliver D. Hart (1986) "The Costs and Benefits of Ownership: A Theory of Vertical and Lateral Integration", Journal of Political Economy, 94:691-719. 